\title{
Oleiculture : Caracterisation De Six Varietes D’olives Introduites Dans Le Sud - Est Algerien
}

\begin{abstract}
Benaziza Abdelaziz
Semad Djamila

Département des sciences agronomiques, Université $\mathrm{M}^{\mathrm{ed}}$ Khider, Biskra (Algérie)

doi: 10.19044/esj.2016.v12n33p537 URL:http://dx.doi.org/10.19044/esj.2016.v12n33p537

Abstract

The cultivation of olive trees in the pre-Saharan areas of Algeria showed yields that are satisfactory and has acceptable values of oil quality. In the context of improvement of culture and order to respond to international norms for a particular exporting oil extra virgin, she should have a lower $0.8 \%$ acidity required by the economic operators.

For enhanced characterization and valorization some local of olive oil varieties introduced, this work falls under this objective and is articulated primarily on six varieties. It is based essentially on oil quality parameters.
\end{abstract}

Keywords: Olive cultivation, characterization, introduced varieties, southeast, Algeria.

\section{Résumé}

La culture de l'olivier dans les zones présahariennes de l'Algérie a montré des rendements qui s'avèrent satisfaisants et une qualité d'huile de valeur acceptable.

Dans l'optique de l'amélioration de la culture et afin de répondre aux normes internationales pour une exportation de l'huile en particulier l'extra vierge, elle doit posséder une acidité inférieure à $0.8 \%$, exigée par les opérateurs économiques.

Pour une meilleure caractérisation et valorisation de certaines variétés d'huile d'olive locales et introduites, ce travail s'inscrit dans cet objectif et s'articule principalement sur six variétés. Il est basé essentiellement sur les paramètres de qualité des huiles.

Mots clés: oléiculture, caractérisation, variétés introduites, sud-est, Algérie. 


\section{Introduction:}

En Algérie, l’oléiculture connaît un essor considérable, la majeure partie est destinée à la production d'huile et à la conservation des fruits. Bien que l'Algérie est considérée parmi les plus producteurs de l'huile d'olive, cette production demeure insuffisante. Pour combler cette efficience, le développement du secteur oléicole revêt une importance primordiale notamment dans le sud du pays.

Dans l'objectif d'étudier les performances de quelques variétés et l'amélioration de la qualité de l'huile dans ces zones et de répondre aux normes internationales pour une meilleure exportation, l'huile algérienne essentiellement extra vierge doit avoir une acidité inférieur à $0.8 \%$. À cet effet, la caractérisation et la valorisation de certaines variétés est nécessaire. Cette étude s'inscrit dans cet objectif et se base sur six variétés d'huile d'olive à savoir Ferkani, Manzanilla, Frantoio, Abani, Tablout et Rougette. Cette caractérisation est fondée sur des paramètres de qualité d'une part (acidité, indice de peroxyde et de l'extinction dans l'UV) et d'autre part sur la composition des huiles en caroténoïdes et en chlorophylles.

\section{Matériel et méthodes:}

\subsection{Matériel végétal:}

Cette étude est réalisée sur six variétés d'huile d'olive, plantées dans la région de Loutaya (Biskra, Algérie), dont quatre variétés sont d'origine algériennes : Ferkani, Abani, Tablout, Rougette et deux variétés étrangères : Frantoio et Manzanilla.

Les olives ayant objet de l'étude ont été récoltées sur des arbres irrigués régulièrement et sont en pleine production. Les échantillons d'olives étudiés et leurs caractéristiques sont illustrés dans le tableau 01.

Les principales analyses sont effectuées au laboratoire de l'institut technique de l'arboriculture et de la vigne (ITAFV de Takrietz, w. Bejaïa). Les démarches exécutées sont indiquées dans le tableau 02. 
Tableau 01 : Caractéristiques des variétés d’olive étudiées.

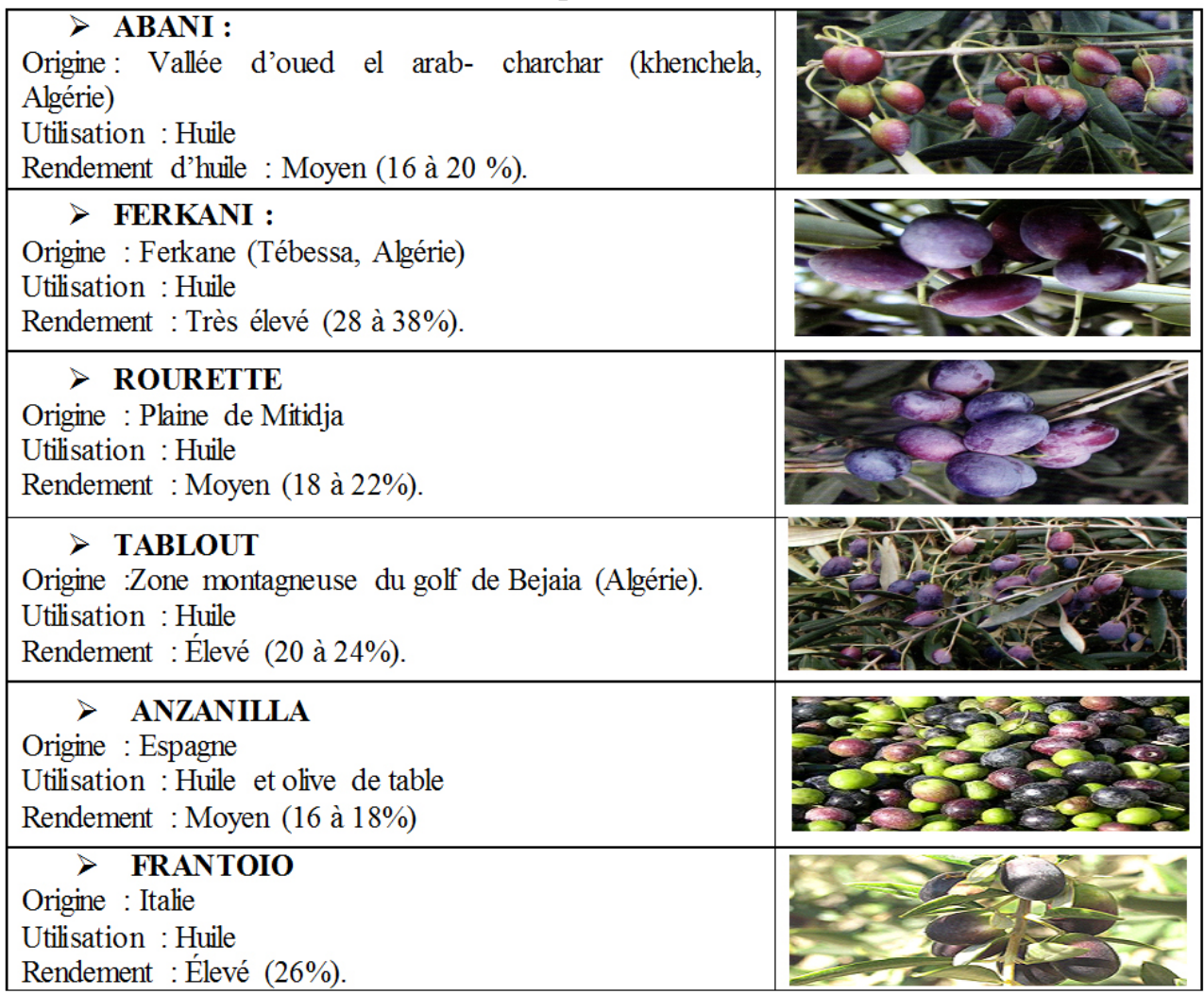

Tableau 02 : Les principales démarches effectuées à la cueillette.

\begin{tabular}{|l|l|l|l|l|l|}
\hline $\begin{array}{l}\text { Stade de } \\
\text { cueillette }\end{array}$ & $\begin{array}{l}\text { Mode de } \\
\text { cueillette }\end{array}$ & $\begin{array}{l}\text { Date } \\
\text { de } \\
\text { récolte }\end{array}$ & $\begin{array}{l}\text { Durée de } \\
\text { stockage }\end{array}$ & $\begin{array}{l}\text { Date de } \\
\text { trituration }\end{array}$ & Système d'extraction \\
\hline $80 \%$ à la véraison & À la main & $\begin{array}{l}15 \\
\text { Nov. }\end{array}$ & 02 jours & 17 nov. & $\begin{array}{l}\text { Oléo } \\
\text { centrifugation }\end{array}$ \\
\hline
\end{tabular}

\subsection{Méthodes:}

\subsubsection{Détermination de l'indice de maturité :}

La détermination de l'indice de maturité a été réalisée conformément à la méthode proposée par le conseil oléicole international en 1984. Il est déterminé par l’appréciation de la couleur du fruit (épiderme et pulpe) d’un échantillon de 100 olives prélevées au hasard, on suivant une classification de 0 à 7 qui correspond aux différentes colorations allant du vert intense jusqu'au noir, ce qui fait distinguer 8 groupes selon les caractéristiques suivantes :

$>$ Groupe 0 : olives à épiderme vert intense ou vert foncé.

$>$ Groupe 1 : olives à épiderme jaune ou vert jaunâtre. 
$>$ Groupe 2 : olives à épiderme jaunâtre présentant des taches ou zones rougeâtre.

$>$ Groupe 3 : olives à épiderme rougeâtre ou violet claire.

$>$ Groupe 4 : olives à épiderme noir et pulpe entièrement verte.

$>$ Groupe 5 : olives à épiderme noir et pulpe violette jusqu'à la moitié de son épaisseur.

$>$ Groupe 6 : olives à épiderme noir et pulpe violette jusqu'au noyau.

> Groupe 7 : olives à épiderme noir et pulpe entièrement foncée.

\subsubsection{Extraction d'huile d'olive:}

Les fruits d'olive ont été triturés en moyen d'un oléo doseur et l'extraction a été faite suivant les étapes suivantes :

Broyage: réalisé dans avec un broyeur à moteur suivi d'un malaxage réalisé en deux étapes ; une à 15 minutes sans eau et l'autre avec $50 \mathrm{ml}$ d'eau tiède à $30 \mathrm{c}^{\circ}$. La centrifugeuse verticale est utilisée pour récupérer l'huile de la pâte dans des flacons en verre fumé, étiquetés et mis au réfrigérateur à $4 c^{\circ}$ afin de les analyser.

\subsubsection{Teneur en eau et en matières volatiles:}

Elle est effectuée conformément à la norme AFNOR NF T60-201, il consiste à provoquer le départ d'eau par introduction d'une quantité connue d'huile dans une étuve maintenue à la température de $103^{\circ} \mathrm{C}$ pendant $30 \mathrm{mn}$.

La teneur en eau et en matières volatiles est calculée selon la formule suivante :

$$
\begin{array}{r}
(\boldsymbol{H} \%)=\frac{\boldsymbol{m} \mathbf{1}-\boldsymbol{m} \mathbf{2}}{\boldsymbol{m} \mathbf{1}-\boldsymbol{m} \mathbf{0}} \mathbf{m}_{\mathbf{1}} \text { : est la masse en gramme de la capsule et de la prise d'essai. } \\
\mathbf{m}_{\mathbf{2}} \text { : est la masse en gramme de la capsule et des résidus après chauffage. }
\end{array}
$$

\subsubsection{Calcul du rendement en huile.}

Il consiste à réaliser l'extraction de l'huile par soxhlet (extraction par solvant) à partir d'une quantité de pâte séchée à $103^{\circ} \mathrm{C}$ pendant quatre heures, en utilisant un solvant approprié (hexane). Le rendement est évalué par la pesée de l'huile obtenue.

$$
\begin{aligned}
R=\left[\left(M-M_{0}\right) / M p e\right] X 100 \text { ou } & \\
& \\
& \mathbf{M}_{0}: \text { : masse en gramme du ballon contenant l'huile, } \\
& \text { Mpe : masse en gramme du ballon vide. }
\end{aligned}
$$

\subsubsection{Détermination de l'absorbance spécifique aux rayonnements ultraviolets.}

Il permet de dissoudre l'échantillon d'huile dans un solvant approprié (cyclohexane) et de déterminer l'absorbance par spectrophotométrie aux 
rayonnements ultraviolets dans un domaine spécifique de longueur d’onde (232nm et $270 \mathrm{~nm})$.

\subsubsection{Acidité:}

C'est le pourcentage d'acides gras libres exprimé conventionnellement en acide oléique de poids moléculaire 282.5g/mole pour l'huile d'olive.

\subsubsection{Indice de peroxyde :}

Cet indice est déterminé après traitement du corps en solution dans de l'acide acétique et du chloroforme par une solution d'iodure de potassium. Le titrage de l'iode libéré par une solution titrée en thiosulfate de sodium.

\subsubsection{La composition en acides gras :}

\section{Préparation des esters éthyliques :}

La prise d'essai (0,2 g d'huile d'olive) est ajoutée à $5 \mathrm{ml}$ d'hexane pour chromatographie et $0,2 \mathrm{ml}$ de solution de $\mathrm{KOH}$ méthanolique $(2 \mathrm{~N})$, le mélange est agité énergiquement pendant $1 \mathrm{~min}$, laissé décanter pendant 15 min, prélevé à l'aide d'une seringue $1 \mathrm{ml}$ du surnageant, puis injecter automatiquement 0,2 $\mu \mathrm{l}$ et démarrer la programmation.

\section{Dosage quantitatif et qualitatif}

Les esters méthyliques sont injectés dans un chromatographe en phase gazeuse dont les conditions d'analyse sont les suivants :

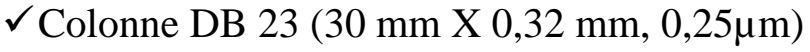

$\checkmark$ Température de l'injecteur (FID): $250^{\circ} \mathrm{C}$

$\checkmark$ Température du détecteur (SPLIT 1/100): $250^{\circ} \mathrm{C}$

$\checkmark$ Débit du gaz vecteur (azote): $1 \mathrm{ml} / \mathrm{min}$

$\checkmark$ Quantité injectée: $0,2 \mu l$

$\checkmark$ Programmation de température: isotherme $\left(190^{\circ} \mathrm{C}\right)$.

\section{$>$ Expression des résultats}

Le corps gras est estérifié en présence de méthanol. Les esters méthyliques d'acides gras sont séparés sur une colonne polaire et sont identifiés en fonction de leur temps de rétention. La surface correspondante à chacun d'eux est calculée et apportée à la surface totale des différents acides gras pour obtenir un pourcentage.

\subsubsection{Détermination de la teneur en composés phénoliques totaux :}

Elle consiste à diluer $0,5 \mathrm{~g}$ d'huile dans $0,5 \mathrm{ml}$ d'hexane et leur ajouter $0,5 \mathrm{ml}$ du mélange méthanol/eau (80/20), centrifuger le mélange pendant 5 minutes à 5000 tours par minute. La phase méthanolique est récupérée. Effectuer une deuxième extraction avec $0,5 \mathrm{ml}$ du méthanol/eau (80/20). A la fin, les deux extraits sont réunis et utilisés pour les analyser. 


\subsubsection{Dosage des O-diphénols}

Le principe est de prendre $0,5 \mathrm{ml}$ d'extrait phénolique, faire dissoudre dans $5 \mathrm{ml}$ de méthanol/eau (1/1), 4ml d'extrait méthanolique sont additionnés de $1 \mathrm{ml}$ de la solution de molybdate de sodium déshydraté à $5 \%$ dans l'éthanol/eau (v/v). Agiter vigoureusement pendant 1 minute, après 15 minutes à l'obscurité, une lecture à $370 \mathrm{~nm}$ est faite. Une courbe d'étalonnage est établie avec l'acide caféique.

\subsubsection{Dosage des chlorophylles :}

Le principe consiste à mesurer l'absorbance des huiles d'olives à 630 $\mathrm{nm}, 670 \mathrm{~nm}$ et $710 \mathrm{~nm}$ en utilisant le tétrachlorure de carbone comme blanc.

\subsubsection{Dosage des caroténoïdes totaux :}

Le principe consiste à mesurer l'absorbance d'1g d'huile dans $9 \mathrm{ml}$ d'hexane à une longueur d'onde de $450 \mathrm{~nm}$ qui correspond au maximum d'absorbance du $\beta$-carotène. Une courbe étalon est établie avec du $\beta$ carotène.

\section{Résultats et discussions:}

\subsection{Indice de maturité:}

Selon ce paramètre, les olives des six variétés cultivées nous ont permis de distinguer 8 groupes:

$>$ Groupe 0 : olives à épiderme vert intense ou vert foncé.

$>$ Groupe 1 : olives à épiderme jaune ou vert jaunâtre.

$>$ Groupe 2 : olives à épiderme jaunâtre présentant des taches ou zones rougeâtre.

$>$ Groupe 3 : olives à épiderme rougeâtre ou violet claire.

$>$ Groupe 4 : olives à épiderme noir et pulpe entièrement verte.

$>$ Groupe 5 : olives à épiderme noir et pulpe violette jusqu'à la moitié de son épaisseur.

$>$ Groupe 6 : olives à épiderme noir et pulpe violette jusqu'au noyau.

$>$ Groupe 7 : olives à épiderme noir et pulpe entièrement foncée.

La maturation de l'olive est un processus lent qui a duré plusieurs mois et varie selon la variété, la disponibilité de l’eau et la température.

Les résultats obtenus pour les différentes variétés sont présentés dans la figure1. 


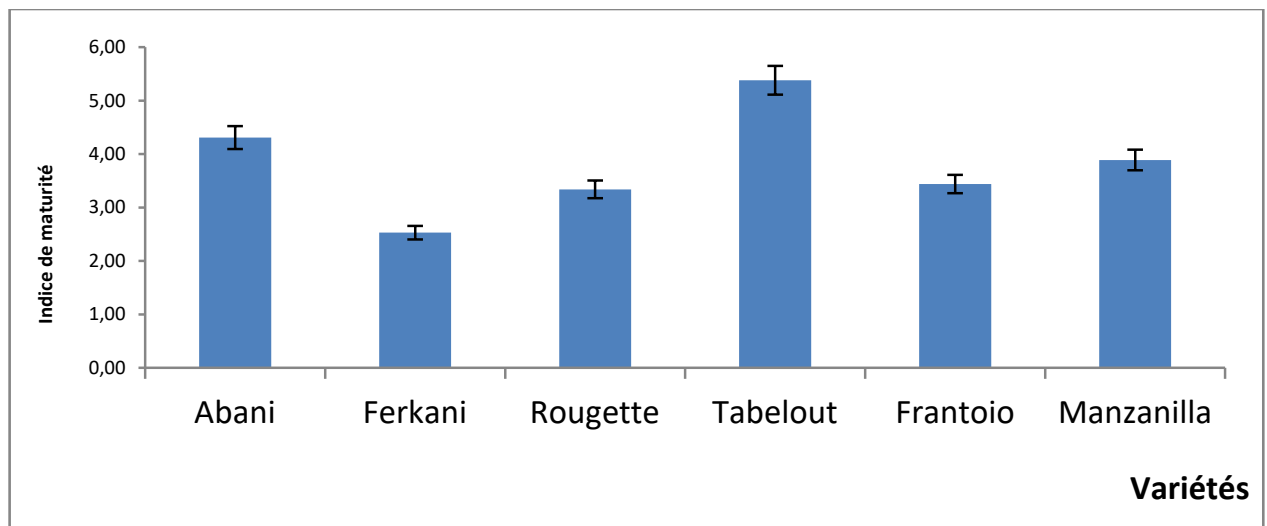

Figure 1 : Les indices de maturité des olives des variétés étudiées

Ce paramètre est d'une manière générale compris entre 2,53 et 5,38 malgré la récolte faite dans la même journée, il varie d'une variété à l'autre (caractère variétal); la variété Tablout se caractérise par une maturité précoce avec un indice de maturité le plus élevé $(5,38)$, suivie de celle d'Abani (4,31). Les variétés Manzanilla et Frantoïo ont des indices de position intermédiaire (3,89 et 3,44 respectivement). Les variétés Rougette et Frantoïo ne présentent pas de différences significatives et la variété Ferkani se démarque des autres variétés par la valeur la plus faible $(2,53)$, ce qui explique la tardivité de cette dernière. Ces constations sont confirmées par El Antari et al., en (2003).

\subsection{Analyse physique:}

\subsubsection{Teneur en eau et matières volatiles:}

Les teneurs moyennes en eau et en matières volatiles des échantillons d'olives des variétés étudiées sont présentées sur la figure 2.

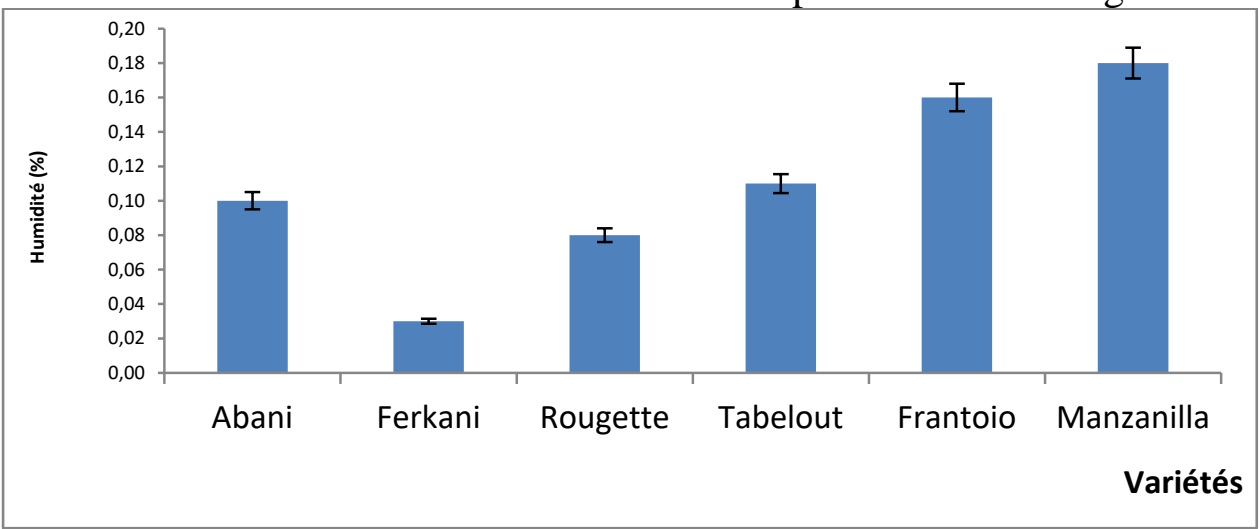

Figure 2 : Teneur en matières volatils des huiles des variétés étudiées. 
Les résultats obtenus pour les six variétés étudiées révèlent que les teneurs en eau et en matières volatiles sont conformes aux normes établies par le comité oléicole international (COI, 2006).

Les variétés Frantoïo et Manzanilla se distinguent par leurs teneurs en humidité légèrement élevées $0,16 \%$ et $0,18 \%$ respectivement, suivies par les variétés Tablout et Abani avec des teneurs spécifiques de 0,11\% et 0,10\%. Les variétés Ferkani et Rougette exhibent les valeurs les plus faibles avec $0,03 \%$ et $0,08 \%$. Cette variation peut être expliquée par l'effet cumulé de la variété et des conditions pédoclimatiques notamment la pluviométrie.

\subsubsection{Rendement en huile au soxhlet:}

Les résultats obtenus pour la teneur en huile sont exprimés en pourcentage de la matière sèche (figure 3 ).

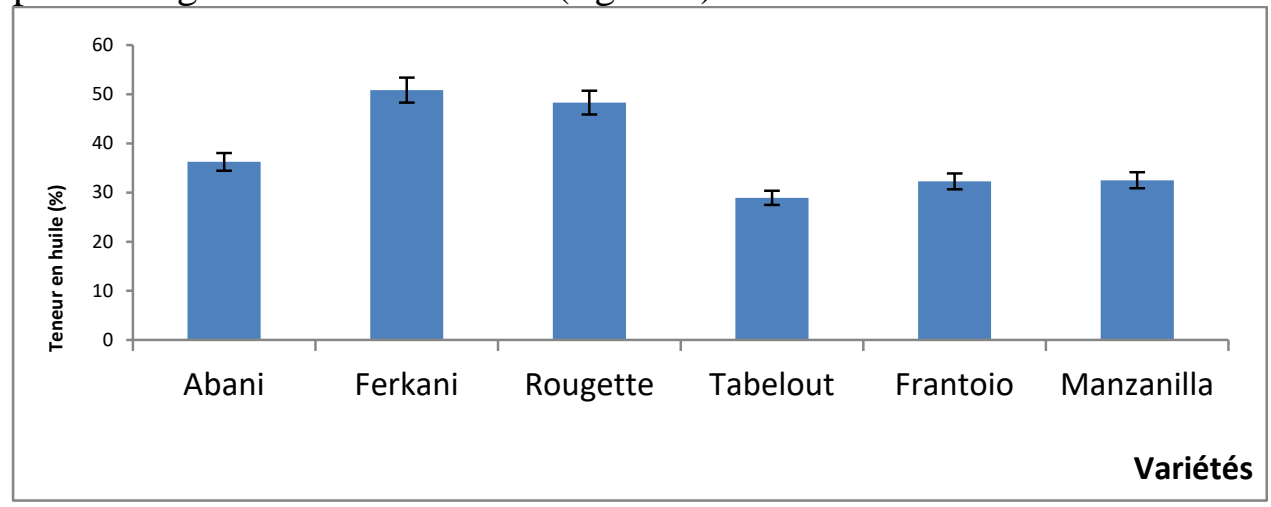

Figure 3 : rendement en huile déterminé au soxhlet.

Ces résultats révèlent une variation du rendement en huile entre 28,92\%, illustrée chez la variété Tablout et 50,87\% chez la variété Ferkani. Ce qui explique l'effet variétal.

\subsubsection{Détermination de l'absorbance spécifique aux rayonnements ultraviolets:}

L'extinction à $232 \mathrm{~nm}$ d'une huile nous renseigne sur son degré d'oxydation (El Antari et al., 2003). Les résultats obtenus sont indiqués dans la figure 4.

Ces résultats concernent les absorbances spécifiques $\left(\mathrm{K}_{232}\right.$ et $\left.\mathrm{K}_{270}\right)$, enregistrées pour les huiles des variétés étudiées et indiquent des différences significatives élevées entre les variétés exception entre Abani et Tablout pour $\mathrm{K}_{270}$.

Les valeurs minimales du coefficient $\mathrm{K}_{232}$ et $\mathrm{K}_{270}$ sont enregistrées chez la variété Rougette (1,02 et 0,07 respectivement) et les valeurs maximales sont notées pour la variété Frontoïo (2,22 et 0,22 respectivement). 
Les échantillons d'huile exhibent des coefficients d'extinctions spécifiques dans l'ultraviolet (K232 et $\mathrm{K}_{270}$ ), inférieurs aux limites établies par le COI (2003) pour une huile d'olive extra vierge $\left(K_{232} \leq 2,5\right.$ et $K_{270} \leq$ $0,22)$.

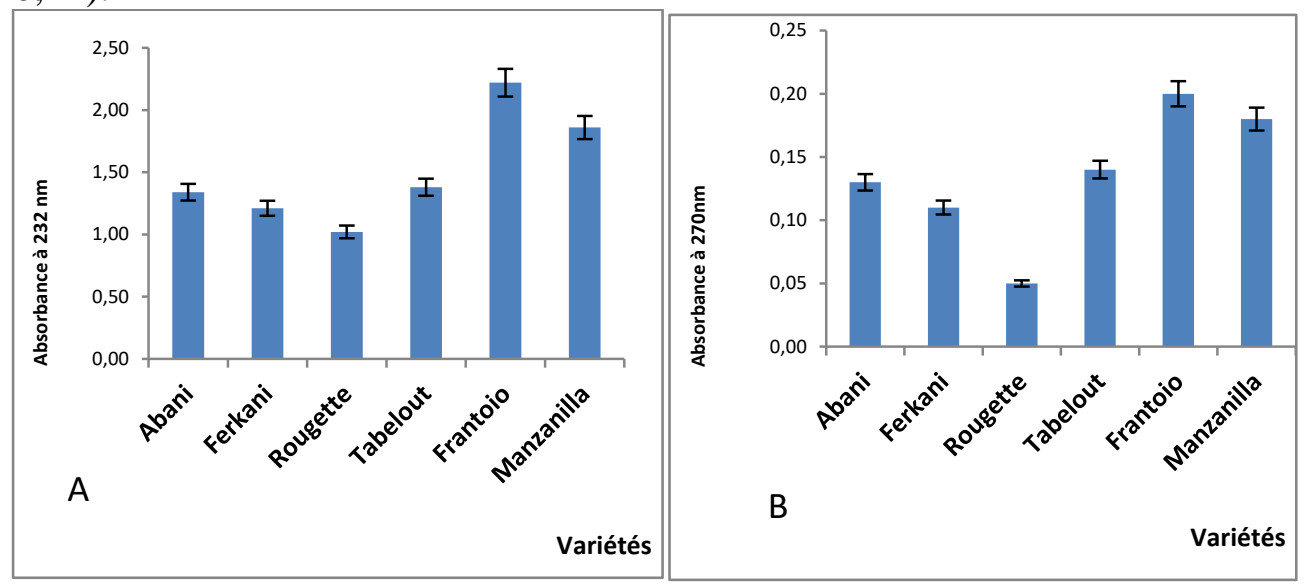

Figure 4 : Coefficient d'absorption spécifique à $232 \mathrm{~nm}$ (A) et à $270 \mathrm{~nm}$ (B).

\subsection{Analyses chimiques:}

\subsubsection{Acidité:}

L'acidité libre est un facteur qui renseigne sur l'altération de l'huile par hydrolyse, c'est un facteur de qualité très important et un critère de classification des huiles d'olive.

Les valeurs d'acidité enregistrées chez les variétés étudiées sont inférieures à $0,8 \%$, ce qui correspond selon le COI (2006) aux huiles d'olive vierge extra.

L'acidité la plus faible est enregistrée chez la variété Tablout (0,25\%), variété a degré de maturité le plus élevé (figure 5).

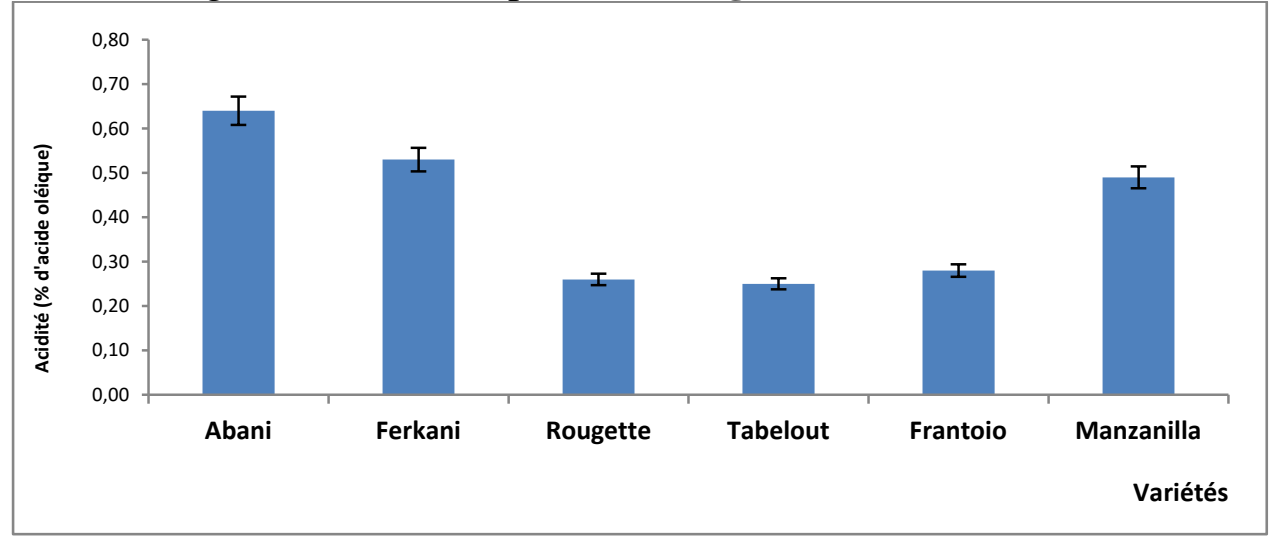

Figure 5 : Acidité libre de l’huile des variétés étudiées. 
Des différences non significatives sont enregistrées entre les variétés Tablout et rougette, entre les variétés Ferkani et Manzanilla et entre Abani et Frantoïo.

Ces résultats sont nettement inférieurs à ceux enregistrés chez les variétés du sud de la Tunisie notamment la variété Chemlali (1\%) et la variété Chétoui (0,9\%) (Issaoui et al., 2010).

\subsubsection{Indice de peroxyde :}

L'indice de peroxyde est lié à la récolte, à la conservation et au mode d'extraction. Il reflète le degré d'oxydation des huiles, accéléré par la présence d'oxygène, la température et certains catalyseurs. Ces facteurs agissent sur les doubles liaisons des acides gras insaturés pour former des peroxydes et des hydro peroxydes (Cimato, 1990).

Les valeurs de l'indice de peroxyde des huiles des variétés étudiées sont illustrées dans la

figure 6.

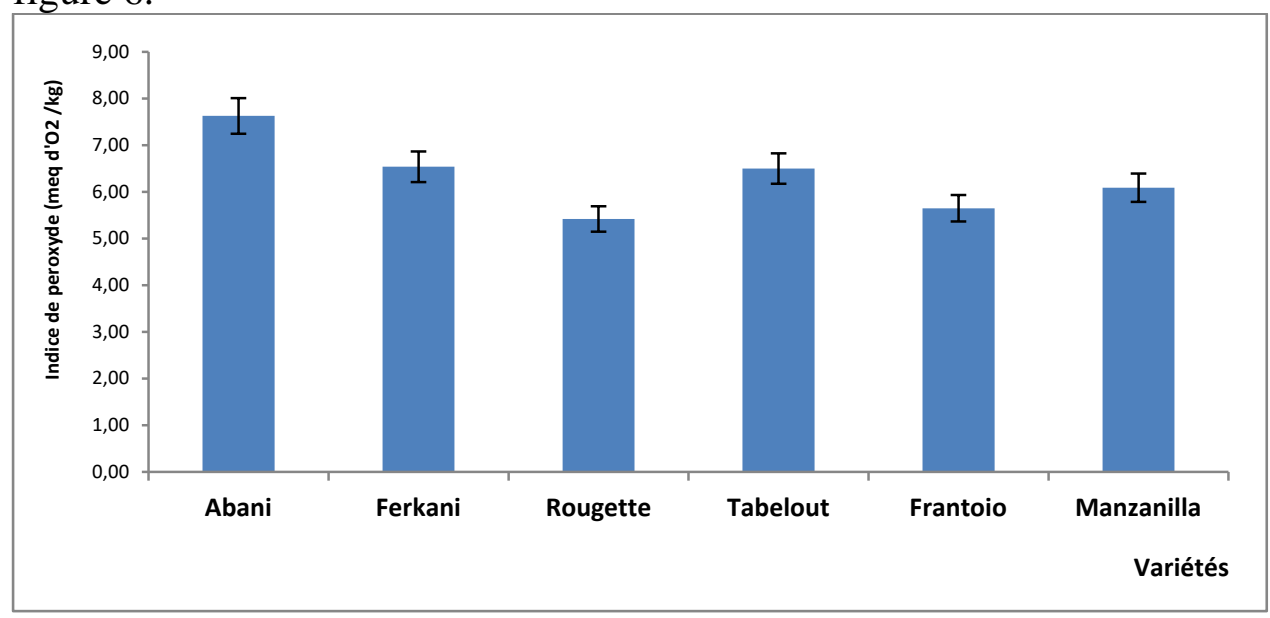

Figure 6 : Indice de peroxyde des huiles des variétés étudiées.

Pour tous les échantillons d'huile étudiés, les valeurs de l'indice de peroxyde enregistrées sont inférieures à 20 meq d'O2/kg, limite fixée par le COI (2003). Ces valeurs sont comprises entre un minimum de $5,42 \mathrm{meq}$ d’O2/kg enregistrée chez la variété Rougette et un maximum de 7,63 meq d’O2/kg chez la variété Abani.

Nos résultats sont nettement inférieurs à ceux rapportés par Torres et Maestri (2006) sur les variétés Arbequira, Ascobra, Manzanilla et Nevodillo, plantées en Argentine dans une zone semi-aride. Ces valeurs de l'indice de peroxyde sont relativement faibles et indiquent une faible oxydation des huiles, cela pourrait être dû aux conditions de transformation des olives (récolte, transport et stockage) et à la présence de substances antioxydantes naturelles. 


\subsection{Analyse de la composition:}

\subsubsection{La composition en acides gras:}

La composition en acides gras totaux est un paramètre de qualité et d'authenticité des huiles. La comparaison des variétés passe obligatoirement par la comparaison de la composition en acides gras (EL Antari et al., 2003).

L'huile d'olive a un profil d'acides gras caractéristiques, dominé par l'acide oléique (C18:1) présent en grande quantité, l'acide linoléique (18:2), l'acide palmitique (C16:1) et l'acide stéarique (C18 :0) (Ryan et al., 1998).

Les résultats de la composition en acides gras des huiles étudiées sont les même sur le plan qualitatif mais ils différent sur le plan quantitatif (figure 7).

Toutes les huiles des variétés étudiées présentent des teneurs en différents acides gras répondant aux normes établies par le COI en 2003 pour les huiles d'olive extra vierge, sauf pour l'acide linoléique qui détient un pourcentage le plus élève que la norme $(<=1 \%)$ pour les variétés Abani (7,25\%), manzanilla (6,81\%), Frantoïo (2,59\%) et Ferkani (3,28\%). Cette variation de la composition en acides gras de l'huile d'olive est essentiellement un caractère variétal (Ryan et al, 1998).

La variété Rougette se démarque des autres variétés par le taux le plus élevé en acide oléique (81,86\%) et le taux le plus faible en acide linoléique (4,86\%), elle est caractérisée ainsi par le rapport acide oléique/acide linoléique le plus élevé (16,91\%). En outre, la variété Frantoïo se caractérise par le taux le plus faible en acide oléique (59,89\%) et le taux le plus élevé en acide linoléique (19,41\%).
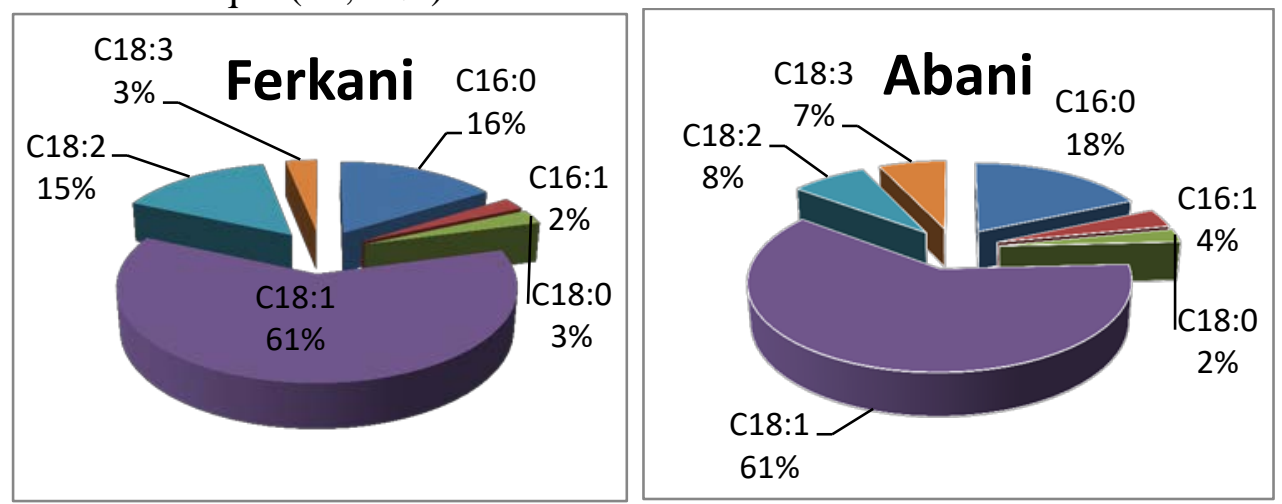

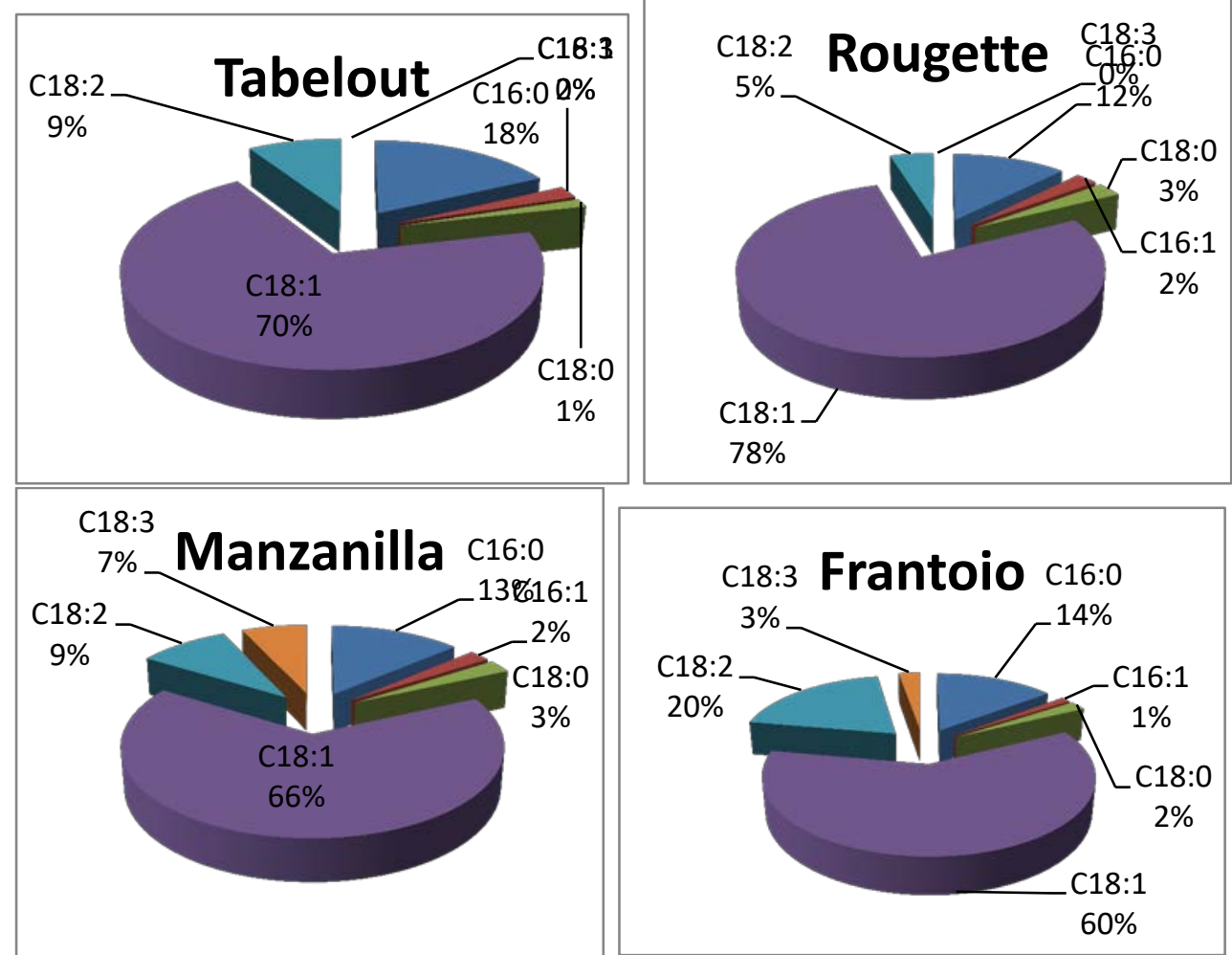

Figure 7 : La composition en acides gras des différentes variétés étudiées.

Après comparaison de ces résultats à ceux obtenus par Torres et Maestri (2006), on constate des similitudes avec la variété Arbequina, ce qui explique que les acides gras polyinsaturés sont obtenus par désaturation de l'acide oléique.

Les variétés Ferkani, Abani, Manzanilla et Tablout se déterminent par des teneurs en acide oléique qui oscillent entre $64,74 \%$ et $69,63 \%$ et des teneurs en acide linoléique qui varient entre $8,17 \%$ et $16,01 \%$, avec un rapport acide oléique / acide linoléique compris entre 3,89 et 7,92. L'acide linolénique (C18:3) est présent avec des quantités très faibles (traces) pour les variétés Tablout et Rougette.

D'après nos résultats, l'acide palmitique (C16:0) se distingue par le taux le plus élevé par rapport aux acides gras saturés des variétés étudiées, son taux est compris entre $12,81 \%$ et $19,26 \%$. La comparaison de nos résultats avec ceux obtenus par EL Antari et al., (2003), relève des similitudes avec les variétés Picholine Marocaine, Picholine Languedoc, Arbequina et Blanquita de Elvas implantées en Tunisie et qui possèdent des taux qui varient entre $10 \%$ et $19 \%$.

L'augmentation du rapport acides gras insaturés/acides gras saturés confère à l'huile d'olive une plus grande stabilité à l'oxydation, elle varie 
entre 4 et 10 selon la variété et la zone géographique, ce qui a été indiqué par Perrin (1992).

D’après nos résultats, ce rapport est compris entre 3,83 et 5,67 , ils sont comparables à ceux rapportés par Torres et Maestri (2006), il varie entre 4,2 et 5,31 pour les variétés Arbequina, Ascolana, Manzanilla et Nivadillo.

\subsubsection{La teneur en composés phénoliques totaux:}

Les composés phénoliques ou polyphénols sont responsables de la bonne stabilité et de l'oxydation des huiles d'olives vierges. En plus des propriétés antioxydantes, ces composés possèdent d'intéressantes propriétés nutritionnelles et organoleptiques.

Selon Ollivier et al., (2004), certains composés phénoliques confèrent aux huiles vierges une saveur amère et une sensation piquante.

La teneur la plus élevée en phénols totaux est enregistrée pour la variété Tablout avec une moyenne de 489,60 ppm et la plus basse pour la variété Manzanilla avec une moyenne de 48,50 ppm. Les autres variétés présentent des teneurs comprises entre 116,50 et 241,33 ppm (figure 8).

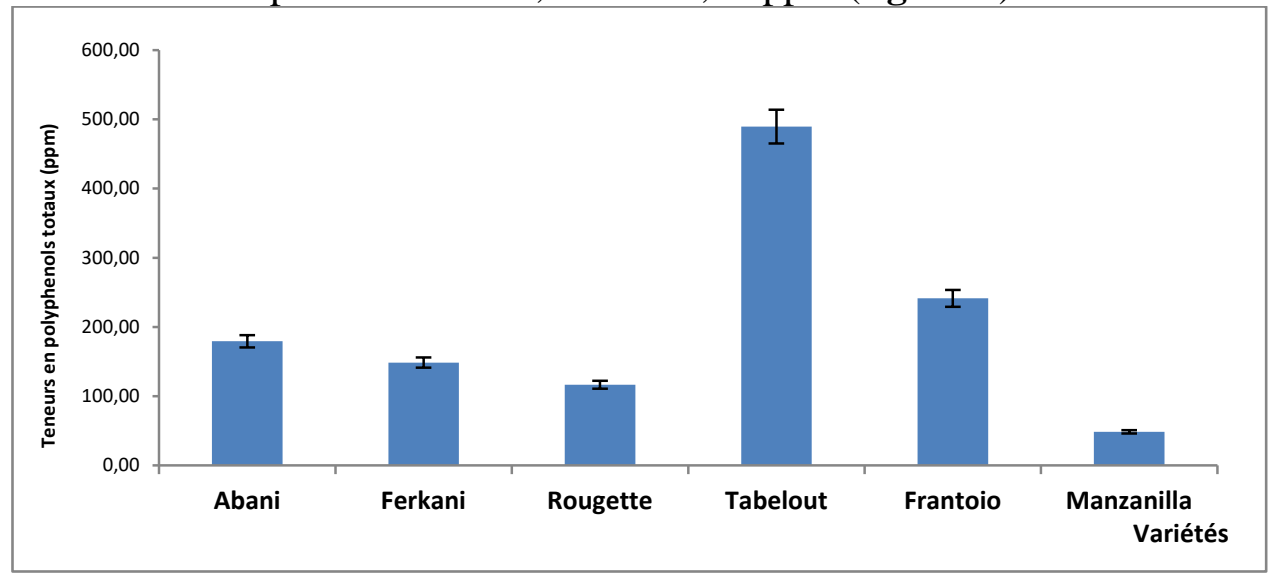

Figure 8 : Teneur en polyphénols totaux des huiles des variétés étudiées.

Les teneurs en polyphénols totaux enregistrées pour nos variétés d’huile sont comparables à celles obtenues par Ranalli et De Mattia (1997).

\subsubsection{Les O-diphénols:}

Les O-diphénols sont les constituants qui confèrent aux huiles d’olive une grande stabilité oxydative (Allalout et al., 2009).

L’analyse des teneurs en O-diphénols, exprimées en milligramme d’équivalent d'acide caféique, les différentes huiles étudiées (figure 9) montrent des différences significatives entre les variétés étudiées. 
D’après les résultats obtenus, il s'avère que la variété Tablout se démarque par le taux le plus élevé en $O$-diphénols (46,74ppm), teneur appréciable et la variété Manzanilla enregistre la valeur la plus faible $(0,68 \mathrm{ppm})$. Les autres variétés ont des valeurs comprises entre 1,60 ppm et 26,83 ppm.

Ces résultats sont nettement inférieurs à ceux rapportés par Allalout et al., (2009), qui oscillent entre 40,88 et 294,46 ppm.

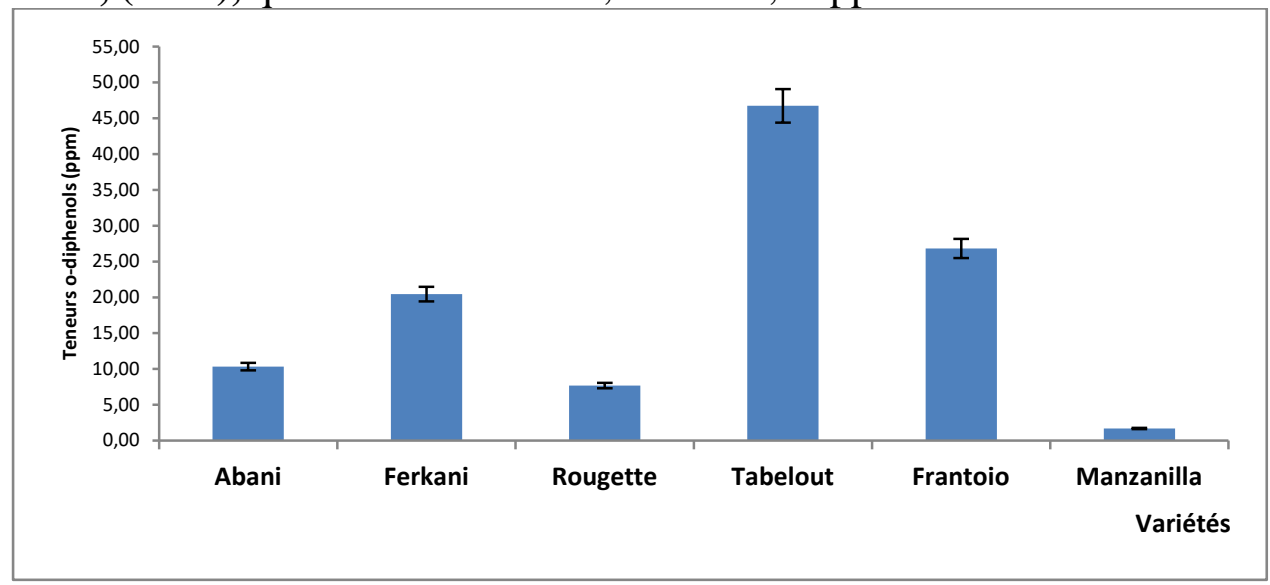

Figure 9 : Teneurs en o-diphénols des huiles des variétés étudiées.

\subsubsection{Les chlorophylles:}

Les chlorophylles sont des pigments responsables de la couleur caractéristique de l'huile d'olive, elles sont impliquées dans les mécanismes d'auto-oxydation et la photo-oxydation (Ryan et al., 1998).

Les teneurs en chlorophylles des huiles des diverses variétés étudiées sont représentées dans la figure 10 .

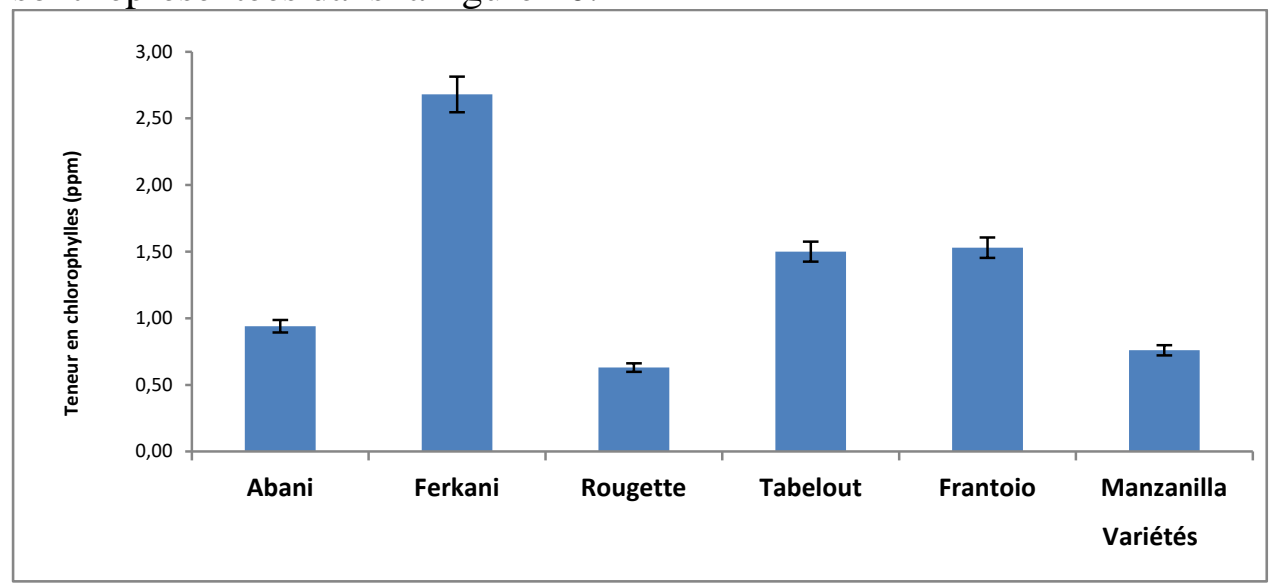

Figure 10 : Teneurs en chlorophylles des huiles des variétés étudiées 
Les résultats obtenus montrent que les teneurs en chlorophylles des huiles testées sont

variables selon les variétés. La quantité la plus élevée est enregistrée pour l'huile issue de la variété Ferkani (2,68 ppm) et la plus faible valeur chez l'huile de la variété Rougette $(0,63 \mathrm{ppm})$. Les teneurs des autres variétés oscillent entre $0,76 \mathrm{ppm}$ et 1,53 ppm.

Les faibles teneurs enregistrées peuvent être dues à l'effet du degré de maturité, le système d'extraction, le sol et les conditions climatiques (Allalout et al. (2009).

En comparant nos résultats à ceux d'Issaoui et al. (2010) obtenus au sud Tunisien, nous constatons que les teneurs en chlorophylles de nos variétés sont largement faibles.

\subsubsection{Les caroténoïdes totaux:}

Les caroténoïdes sont des substances antioxydantes naturelles liposolubles jouant un rôle de pigment de couleur jaune à rouge dans beaucoup de fruits et légumes dont le plus connu est le $\beta$-carotène (Nève, 2002).

D’après les résultats obtenus (figure 12), on constate des différences entre les échantillons d’huiles étudiées, néanmoins, aucune différence n’est observée entre les variétés Ferkani, Abani et la Frantoïo et entre les variétés Ferkani, Abani et Manzanilla.

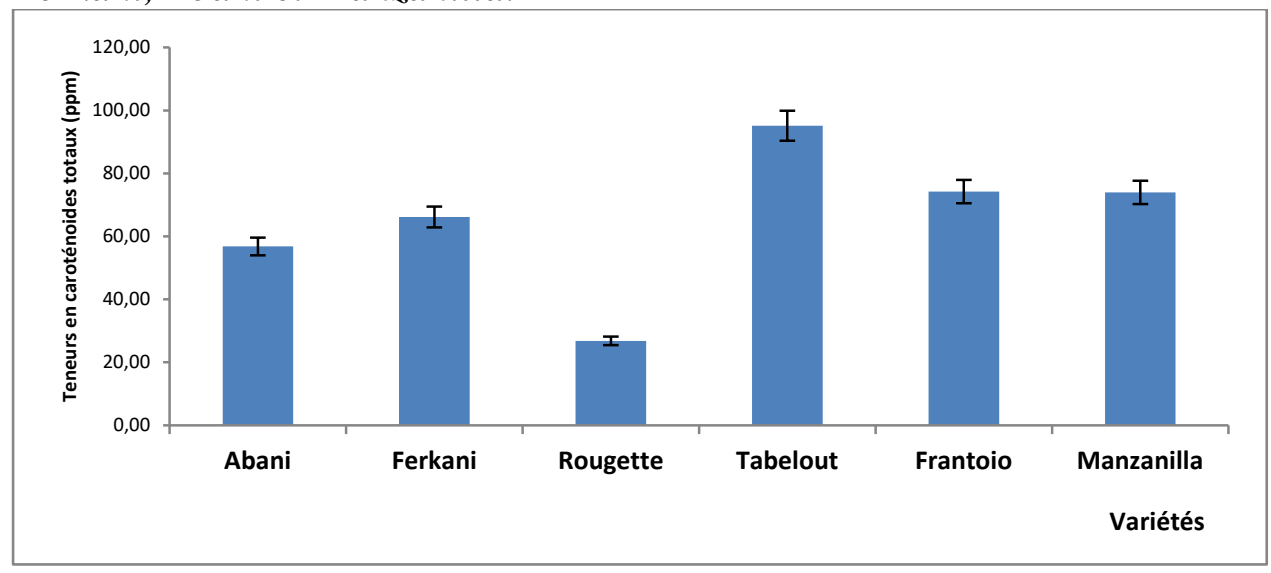

Figure 11 : Teneur en caroténoïdes totaux des huiles des variétés étudiées.

Selon les résultats obtenus par DABBOU et al., (2010) dans les zones arides de la Tunisie pour les variétés Arbequina (11,99ppm), Coratina (16,05 ppm), Koroneiki (20,03 ppm), Chemlali Boughrara (11,40 ppm), Chemlali Zarzis (17,49 ppm), et Chemlali Sfax (6,58 ppm), nous constatons que nos valeurs sont nettement supérieures . 


\section{Conclusion:}

Ce travail permet d'évaluer les caractéristiques physico-chimiques de six variétés, introduites dans la région de Biskra (sud-est d’Algérie) dans le cadre de l’extension oléicole dans les zones subsahariennes.

L'ensemble des résultats d'analyses effectués sur les différents échantillons d’huile d’olive rejoignent les normes fixées par le C.O.I relative à la catégorie des huiles d'olive vierge extra.

La caractérisation pomologique des différentes variétés étudiées a révélé que l'indice de maturité et le rendement en huile des olives sont des paramètres déterminants des variétés.

Concernant la composition en acides gras, les huiles présentent des teneurs différentes avec une dominance particulière de l'acide oléique. Les cultivars Abani, Frantoio, Ferkani et Manzanilla ont enregistré des teneurs élevées en acide linoléique, qui dépassent les normes du COI (2009).

Relativement aux composés phénoliques, les teneurs sont appréciables (>100 ppm) pour cinq variétés et la variété Manzanilla se démarque par une teneur faible (48,50ppm). Cette richesse en composés phénoliques des huiles étudiées joue un rôle capital dans la flaveur et la stabilité des huiles.

Ces données préliminaires forment la base de sélection des variétés pour les futures plantations dans la région. En effet, ces variétés montrent une certaine adaptation aux conditions pédoclimatiques de la zone et présentent des rendements satisfaisants, parmi celles-ci :

La variété Tablout qui semble la plus performante, elle se distingue par des teneurs élevées en composés mineurs (polyphénols totaux, Odiphénols et caroténoïdes), elle montre ainsi une meilleure activité antioxydante et une stabilité au cours de la conservation.

$>$ La variété Rougette se distingue par une teneur élevée en acide oléique $(81,86 \%)$

$>\quad$ La variété Ferkani présente un rendement en huile le plus élevé (50,87\%) et des teneurs appréciables en acide oléique et en composés mineurs.

Ces premiers résultats constituent le point focal du développement de l'oléiculture et des perspectives dans le sud algérien et d'évaluer la qualité des huiles obtenues et de répondre aux exigences du marché national et international et de parvenir aux appellations d'origines contrôlées des huiles.

\section{References:}

1. Boskou D. Characteristics of the Olive Tree and Olive Fruit. In «Olive Oil Chemistry and Technology». 2006. 2 ème Edit. (2): pp1319.

2. El Antari A., El Moudni A. et Ajana H. Évolution comparative de la qualité et de la composition acidique de l'huile d'olive chez 
quelques variétés méditerranéennes cultivées au Maroc. Oliveae. 2003. (95). pp 26-31.

3. COI. L’oléiculture en Algérie. Conseil Oléicole International. 2006. (32). pp 2-22.

4. Issaoui M., Flamini G., Brahmi F., Effect of the growing area conditions on differentiation between Chemlali and Chétoui olive oils. Food Chemistry. 2010. (119). pp 220-225.

5. CIMATO A. La qualité de l'huile d'olive et facteurs agronomiques. Olivae, 1990 (31). pp : 20-31.

6. Torres M. et Maestri M. The effect of genotype and extraction methods on chemical composition of virgin olive oil from Traslasierra Valley (Cordoba, Argentina). Food Chemistry. 2006. (96). pp 507-511.

7. Ryan D., Robards K. et Lavees S. Évaluation de la qualité de l'huile d'olive. Olivae 1998. (72). pp 23-38.

8. COI. Dénominations et définitions des huiles d'olive et des huiles de grignons d'olive.Conseil Oléicole International/7-88-IV/2003. Madrid 2003. pp 46-48.

9. COI., La production et la consommation d'huile d'olive à l'horizon 2010 en Algérie. Revue Olivea. (99). 2003. 56p.

10. Perrin J. Les composés mineurs et les anti oxygènes naturels de l'olive et de son huile. Revue Française des Corps gras. (39) 1992. pp 25-31.

11. Ollivier D., Baubault E., Pinatel C., Souillol S., Guérère M. et Artaud J., Analyse de la fraction phénolique des huiles d'olive vierges. In « Annales des falsifications, de l'expertise et toxicologie, 2éme Semestre (965) 2004. pp 169-196.

12. Ranalli A. et De Mattia G. Characterisation of Olive Oil Produced with a New Enzyme Processing Aid. J Am Oil Chem Soc. 74 (9) 1997. pp 1105-1113.

13. Allalout A., Krichène D., Methenni K., Taamalli., Oueslati I., Daoud D. et Zarrouk M. Characterization of virgin olive oil from intensive Spanish and Greek varieties grown in northern Tunisia. Scientia Horiculture: 2009. pp 77-83.

14. Néve J. Modulation de l'apport alimentaire en antioxydant. Optimisation of dietary intake of antioxydant. Nutrition clinique. (36 ). 2002. pp 292-300. 